\title{
Oficinas de construção de indicadores e dispositivos de avaliação: uma nova técnica de consenso
}

\section{Workshops for the construction of indicators and evaluation devices: a new technique for consensus}

\section{Rosana Teresa Onocko Campos*}

Professora do Departamento de Medicina Preventiva e Social da Faculdade de Ciências Médicas, Universidade Estadual de Campinas UNICAMP, Campinas, SP, Brasil

\section{Lilian Miranda**}

Doutora em Saúde Coletiva pelo Departamento de Medicina Preventiva e Social da Faculdade de Ciências Médicas, Universidade Estadual de Campinas - UNICAMP, Campinas, SP, Brasil

\section{Carlos Alberto Pegolo da Gama***}

Doutorando em Saúde Coletiva pelo Departamento de Medicina Preventiva e Social da Faculdade de Ciências Médicas, Universidade Estadual de Campinas - UNICAMP, Campinas, SP, Brasil

\section{Ana Luiza Ferrer****}

Doutoranda pelo Departamento de Medicina Preventiva e Social da Faculdade de Ciências Médicas, Universidade Estadual de Campinas UNICAMP, Campinas, SP, Brasil

\section{Alberto R. Diaz*****}

Doutorando em Saúde Coletiva pelo Departamento de Medicina Preventiva e Social da Faculdade de Ciências Médicas, Universidade Estadual de Campinas - UNICAMP, Campinas, SP, Brasil

\section{Laura Gonçalves******}

Doutoranda pelo Departamento de Medicina Preventiva e Social da Faculdade de Ciências Médicas, Universidade Estadual de Campinas UNICAMP, Campinas, SP, Brasil

\section{Thiago Lavras Trapé*******}

Mestrando em Saúde Coletiva pelo Departamento de Medicina Preventiva e Social da Faculdade de Ciências Médicas, Universidade Estadual de Campinas - UNICAMP, Campinas, SP, Brasil 


\title{
RESUMO
}

Este artigo discute o trabalho com Oficinas de construção de indicadores e dispositivos de avaliação enquanto uma nova técnica de consensos, especialmente no contexto de uma pesquisa participativa e avaliativa. Para tanto, apresenta as tradicionais técnicas de consenso, bem como o método de Oficinas, trabalhado pelo Grupo de Pesquisa Saúde Coletiva e Saúde Mental: interfaces. Conclui-se que as Oficinas apresentam vantagens frente às tradicionais técnicas de consenso na medida em que aumentam a diversidade dos atores e as inserções sócio-políticas dos participantes, não se restringindo apenas àqueles reconhecidos como especialistas no objeto estudado. A inclusão desses diferentes atores na construção do conhecimento alinha-se à aposta no protagonismo como fator decisivo na incorporação dos resultados do processo avaliativo no cotidiano do trabalho. No entanto, exige infra-estrutura para o encontro presencial entre os participantes e habilidade para lidar democraticamente com as diferentes posições de poder nelas incluídas.

Palavras-chave: Pesquisa qualitativa, Oficina de trabalho para consenso, Avaliação de programas e projetos de saúde e pesquisa participativa baseada na comunidade.

\begin{abstract}
This article discusses the work with Workshops for the construction of indicators and evaluation devices as a new technique of consensus, especially in the context of a participatory and evaluative research. To do so, it presents the traditional techniques of consensus, as well as the method of Workshops, used by the Research Group of Public Health and Mental Health: interfaces. It is concluded that the workshops present ad-vantages in front of the traditional techniques of consensus in that it increases the diversity of actors and the socio-political insertions of participants, not restricting itself only to those recognized as specialists in the subject studied. The inclusion of these different actors in the construction of knowledge aligns with betting on protagonism as a decisive factor in the role of incorporating the results of the evaluation process in the daily work. However, it demands infrastructure for the presential meeting between the participants and skill to deal democratically with the different positions of power therein contained.

Keywords: Qualitative research, Consensus development conferences, Program evaluation, Community-based participatory research.
\end{abstract}

\section{I ntrodução}

O panorama do desenvolvimento de pesquisa na área da saúde se apresenta fortemente marcado pelo predomínio de técnicas quantitativas e, na maioria dos casos, vinculadas a uma base epistemológica que aposta na neutralidade do pesquisador e nas análises estatísticas como única possibilidade de acesso ao conhecimento. No campo da saúde mental, em virtude da própria complexidade do objeto, muitas pesquisas têm lançado mão de metodologias qualitativas que possuem base epistemológica bastante diferente da tradicional. Nas pesquisas que temos desenvolvido, propomo-nos a fazer uma análise crítica do próprio processo de pesquisa, procurando entender o lugar do pesquisador, as relações de 
poder inerentes ao processo, a relação da pesquisa com os demais atores sociais e, em última instância, as possíveis contribuições da pesquisa para a comunidade em geral.

É a partir deste cenário que desejamos refletir sobre algumas experiências desenvolvidas pelo grupo "Saúde Coletiva e Saúde Mental: Interfaces"1, utilizando metodologias de pesquisa qualitativas e participativas. O grupo foi criado em 2004 no Departamento de Medicina Preventiva e Social da Unicamp e desde sua fundação vem desenvolvendo pesquisas relacionadas ao campo da Saúde Mental no contexto das Políticas Públicas. Temos salientado a complexidade do tema da Saúde Mental, vinculado a uma série de questões de natureza sociais, políticas e éticas. O entendimento de que a produção de saúde está diretamente relacionada com a produção de subjetividade (CAMPOS, 2000; ONOCKO CAMPOS, 2001) coloca-nos uma série de desafios enquanto pesquisadores.

O trabalho que vem sendo realizado pelo grupo possui algumas características que merecem ser destacadas, pois faz parte da construção de um estofo epistemológico que considera a interação e reconstrução mútua entre objeto, investigador e realidade, o que influencia profundamente a metodologia de trabalho, balizando o tipo de conhecimento almejado. A primeira característica relaciona-se a uma tradição do Departamento de Medicina Preventiva e Social da FCM/Unicamp de desenvolver seus trabalhos em parceria com a rede pública de saúde. Esta proximidade permite amplo acesso dos pesquisadores ao campo da saúde pública local e o estabelecimento de relações de cooperação profícuas tanto para os serviços quanto para a Universidade.

Outra característica própria ao nosso grupo é a fundamentação que temos dado ao trabalho a partir da hermenêutica crítica (GADAMER, 1997; ONOCKO CAMPOS, 2005), como postura metodológica que nos orienta na efetivação de pesquisas em consonância com a chamada Quarta Geração de Avaliadores, ou Pesquisa Avaliativa Participativa Pluralista (stakeholder-based evaluation). Assim, enfocamos a avaliação como processo participativo e inclusivo, visando à detecção e à consideração das percepções, valores e questões dos grupos de interesse envolvidos com um dado programa, bem como incluindo o maior número de atores no processo, sejam usuários, familiares, gestores e outros que tenham interesse na utilização efetiva dos resultados do processo avaliativo (GUBA; LINCOLN, 1989; FURTADO, 2001).

A afirmação da importância da inclusão de diferentes grupos de interesse no processo avaliativo torna necessária a construção de metodologias de pesquisa que sejam coerentes com nosso quadro de referências. Ao assumirmos uma postura ético-política de garantia da participação de diferentes atores nas diversas fases da pesquisa, somos convocados à necessidade de criação de espaços de reflexão e 
também de devolutivas dos materiais elaborados nas pesquisas, com o objetivo de envolver os grupos de interesse nas problemáticas em foco e ampliar conjuntamente o conhecimento através do processo avaliativo. Destacamos nosso interesse em trabalhar de modo tal que os atores envolvidos no serviço participem da avaliação e possam continuar utilizando os resultados da pesquisa, após a sua conclusão (FURTADO; ONOCKO CAMPOS, 2008).

O objetivo das pesquisas que temos desenvolvido é justamente ajudar na avaliação das práticas de saúde no SUS, colaborando com a avaliação do impacto da implantação das políticas públicas da área de saúde mental (CAMPOS, 2008). Temos buscado compreender como está se dando a transposição das diretrizes preconizadas pelas políticas públicas para a assistência e a gestão dos serviços, extraindo conseqüências práticas do processo e propondo alternativas, visando a melhorias (FURTADO; ONOCKO CAMPOS, 2005).

\section{Objetivo}

No presente trabalho pretendemos refletir a respeito de algumas contribuições produzidas a partir da realização da "Pesquisa avaliativa de uma rede de centros de atenção psicossocial (CAPS): entre a saúde coletiva e a saúde mental" ${ }^{2}$. Focaremos nossa discussão na construção de um dispositivo da pesquisa nomeado de Oficina de construção de indicadores e dispositivos de avaliação, tomado por nós como uma estratégia para a produção de consensos a respeito de quais seriam os indicadores mais adequados para se avaliar um CAPS. Este dispositivo teve como inspiração algumas técnicas tradicionais que são usadas para a construção de consensos, no entanto, percorreu caminhos muito diferentes, na medida em que buscou ser coerente com a aposta epistemológica do grupo.

Nosso objetivo central é discutir a Oficina de construção de indicadores e dispositivos de avaliação, tal como a formulamos, como uma nova técnica de consensos. Objetivamos ainda discutir as vantagens e limitações dessa técnica em relação à Técnica Delphi e aos Grupos nominais, bem como apontar as convergências entre os resultados obtidos nas oficinas e os pressupostos epistemológicos que norteiam nossos trabalhos de pesquisa.

Para realizar tal reflexão, iniciaremos por apresentar a pesquisa avaliativa que deu origem a tais Oficinas. Em seguida, faremos uma breve revisão das tradicionais técnicas de consenso, para então demonstrar o modo como concebemos e experimentamos as Oficinas.

\section{A pesquisa avaliativa de uma rede de centros de atenção psicossocial: entre a saúde coletiva e a saúde mental}

Conduzida pelo grupo de pesquisa "Saúde Coletiva e Saúde Mental: interfaces", em parceria com pesquisadores do grupo de estudos do 
Departamento de Psicologia da Universidade Federal Fluminense (UFF), a Pesquisa avaliativa de uma rede de centros de atenção psicossocial (CAPS): entre a saúde coletiva e a saúde mental objetivou analisar os principais aspectos inerentes à rede de CAPS III do município de Campinas $^{3}$, avaliando os modelos assistenciais, de gestão e de formação dos trabalhadores dos CAPS.

As questões que deram origem a esse projeto de pesquisa foram oriundas da proximidade e participação dos pesquisadores principais com a rede de serviços de saúde mental das cidades de Campinas e Rio de Janeiro e do compromisso com a reforma sanitária e psiquiátrica brasileira. Apoiados na hermenêutica-crítica (RICOEUR, 1990; GADAMER, 1997) como postura metodológica no desenvolvimento dessa pesquisa, fomos a campo para a construção do material empírico, utilizando a técnica de grupos focais adaptada às exigências de nossos objetivos de pesquisa e da conformação do campo empírico (MIRANDA , L.; FIGUEIREDO, M. D. ; FERRER, A. L. ; ONOCKO CAMPOS, R. T. 2008).

No total, realizamos 20 grupos focais, divididos em duas etapas. A composição dos grupos seguiu como critério de homogeneidade a inserção institucional dos envolvidos: um grupo composto com os trabalhadores da assistência de cada um dos seis serviços pesquisados, um grupo com os gerentes dos CAPS III, um grupo com o colegiado municipal de saúde mental, um grupo envolvendo usuários dos seis serviços e um grupo envolvendo familiares de usuários dos seis serviços. Estes 10 grupos participaram de duas etapas de grupos focais. Houve uma grande preocupação em garantir efetivamente a participação de todos os envolvidos, buscando evitar "o risco de negligenciar perspectivas legítimas" (FURTADO; ONOCKO CAMPOS, 2008, p.190).

Para o tratamento das informações coletadas em campo, utilizamos a abordagem narrativa (RICOEUR, 1994). Assim, a partir da transcrição de cada grupo focal gravado construímos uma narrativa (ONOCKO CAMPOS; FURTADO, 2008) utilizando uma metodologia em que o agenciamento dos fatos foi encadeado por um ordenamento lógico, deixando claras as argumentações sobre os temas debatidos, assim como os consensos e divergências de cada grupo. Na segunda etapa dos grupos focais, os mesmos grupos foram reunidos e as narrativas produzidas a partir do primeiro grupo foram devolvidas aos participantes, que puderam discuti-las, contestá-las, validá-las e aprofundá-las. Após a transcrição dos grupos desta segunda rodada, também construímos narrativas referentes a cada um deles.

A análise de todo o material de campo consistiu no agrupamento de núcleos narrativos ${ }^{4}$ identificados nas argumentações em torno dos eixos temáticos de interesse da pesquisa constituindo o que nomeamos de "grades de interpretação". 
Os temas, pré-definidos pelos pesquisadores e contemplados pelos roteiros dos grupos focais, foram: concepção de CAPS, modelos de gestão, formas de abordagens à crise, práticas grupais, Projeto Terapêutico Individual, formação profissional e sofrimento dos trabalhadores, relações com a Rede. Nas grades de interpretação, condensamos os principais argumentos emitidos pelos participantes dos grupos, o que nos permitiu visualizar como cada grupo posicionava-se em relação a cada tema. Em um segundo momento, agrupamos os argumentos de todos os grupos, relativos a cada tema abordado (núcleos argumentais), o que nos permitiu visualizar como - conjunto de atores envolvidos na pesquisa se posicionava frente às temáticas debatidas ${ }^{5}$. As discussões realizadas nas duas etapas dos grupos focais produziram um material rico e heterogêneo sobre questões intrínsecas à organização e funcionamento do cotidiano dos serviços pesquisados.

Passamos para a fase seguinte da pesquisa, com a realização das Oficinas, conforme relataremos mais adiante. Entendendo que as Oficinas, tal como as concebemos, podem funcionar como estratégia de produção de consensos, apresentaremo-nas após uma breve revisão das duas técnicas tradicionais de construção de consenso.

\section{Uma tradição: as técnicas de construção de consenso}

Para a coleta de dados em pesquisas qualitativas e avaliativas em saúde, comumente são utilizados questionários, entrevistas, observações e grupos focais. As técnicas de construção de consensos são utilizadas, em geral, quando não existe unanimidade de opinião sobre determinado assunto, seja porque não existem dados relacionados ao tema, ou porque os dados existentes são controversos entre si.

Costumam ser aplicadas para resolver, por exemplo, incoerências entre resultados de estudos publicados e as suas potencialidades, sendo mais bem aproveitadas na avaliação qualitativa das evidências (JONES; HUNTER, 1995). No campo da saúde, são utilizadas tanto para a prática clínica como para problemas em serviços de saúde (JONES; HUNTER, 1995, 2005; MURPHY M. K.; BLACK, N. A.; LAMPING, D. L.; MCKEE, C. M.; SANDERSON, C. F.; ASKHAM, J.; MARTEAU, T. 1998).

As duas técnicas mais utilizadas são a Técnica Delphi e a Técnica de Grupo Nominal (TGN, também conhecida como painel de especialistas). Ambas avaliam a extensão da concordância (medida de consenso), sendo que a TGN também serve para resolver as discordâncias, possibilitando 0 desenvolvimento de consenso (MURPHY M. K.; BLACK, N. A.; LAMPING, D. L.; MCKEE, C. M.; SANDERSON, C. F.; ASKHAM, J.; MARTEAU, T. 1998; JONES; HUNTER, 2005). 


\section{Técnica de Grupo Nominal}

A Técnica de Grupo Nominal (TGN) foi desenvolvida na década de 1960, nos Estados Unidos, e tem sido utilizada em diversas áreas (serviço social, educação, governo, indústrias). Na área da saúde tem sido utilizada para diversos campos de prática, sempre no sentido de gerar consenso para a tomada de decisões (JONES; HUNTER, 1995).

O termo nominal refere-se a processos que reúnem os indivíduos, mas prescinde do debate entre eles. A TGN foi elaborada inicialmente com a finalidade de aumentar a produtividade do grupo, facilitar a decisão grupal, estimular a geração de idéias críticas e servir como instrumento no agrupamento de idéias (CASSIANI; RODRIGUES, 1996). Tal técnica também permite, na opinião de alguns autores, a validação qualitativa de evidências (JONES; HUNTER, 1995, 2005; MURPHY, M. K.; BLACK, N. A.; LAMPING, D. L.; MCKEE, C. M.; SANDERSON, C. F.; ASKHAM, J.; MARTEAU, T. 1998).

Essa técnica é uma variante estruturada dos métodos para discussão em grupos pequenos e, nesse sentido, ao evitar que uma pessoa domine o debate, estimula a participação daqueles mais passivos. Às vezes também chamada de Painel de Especialistas, compreende um processo que, recorrendo a um grupo de especialistas no assunto, permite selecionar, fazer julgamentos e fomentar a criatividade de sugestões para a resolução de um problema (MENDES, W.; TRAVASSOS, C.; MARTINS, M.; MARQUES, P. M. 2008). Estruturado para a geração de idéias coletivas por meio do incentivo à participação, permitindo a interação dos diversos saberes envolvidos no assunto, visa à geração de consensos, possibilitando assim criar compromisso com o material produzido coletivamente, principalmente quando o objetivo é identificar e propor estratégias de ação.

O encontro para o Grupo Nominal está estruturado da seguinte forma: os participantes (de 9 a12) têm alguns minutos para escrever as suas opiniões sobre o tema em questão; cada participante, assim, contribui com uma idéia para o facilitador, que a registra em algum lugar visível (lousa, cartaz); sugestões semelhantes são agrupadas e, se necessário, é feito um debate grupal, só para esclarecer cada idéia; cada participante, individualmente e, em privado, pondera cada idéia (1a. Rodada); o ranking é tabulado e apresentado; a classificação geral é discutida e é realizada uma nova ponderação (2a. Rodada); as classificações finais são tabuladas e é feita uma devolução dos resultados para os participantes (JONES; HUNTER, 1995). A TGN não é recomendável quando o objetivo é obter uma série de idéias sobre algum tópico, ou fazer uma análise qualitativa de algum processo grupal.

\section{Técnica Delphi}

A técnica Delphi tem seu nome inspirado no deus grego Apolo, um mestre da cidade de Delphi, que tinha habilidades reconhecidas na 
arte de predizer o futuro (JONES; HUNTER, 1995). Disseminada no começo dos anos 60 , foi inicialmente concebida como instrumento de previsão sobre temas internacionais e militares e posteriormente como procedimento de predição no campo empresarial, de novas tecnologias, na sociologia e na saúde (PIOLA; VIANNA; VIVASCONSUELO, 2002). Tem como objetivo a busca de opiniões coletivas qualificadas, consensos de opiniões de um grupo de especialistas a respeito de eventos futuros, sendo os consensos entendidos como a consolidação do julgamento intuitivo do grupo (WRIGHT; GIOVINAZZO, 2000).

A operação da Técnica baseia-se em um processo em que um questionário (cuidadosamente elaborado, apresentando para cada questão uma síntese das principais informações sobre o assunto) circula repetidas vezes entre um grupo de especialistas previamente selecionados, sem que eles se encontrem pessoalmente. Na primeira rodada do questionário são apresentadas questões que requerem respostas quantitativas, com espaço para as justificativas. $\mathrm{Na}$ segunda rodada, são apresentadas as tabulações dessas respostas com tratamentos estatísticos simples e a associação de argumentos com os dados numéricos. A cada nova rodada, as perguntas são repetidas e os participantes devem elaborar suas respostas com base nos resultados estatísticos e nas justificativas às respostas anteriores (reunidas na nova rodada). Esse processo repete-se até que as divergências de opiniões tenham diminuído a um nível satisfatório e a resposta da última rodada possa ser considerada como representativa do consenso do grupo (WRIGT; GIOVINAZZO, 2000).

Para operar desse modo, a Técnica Delphi possui três condições básicas de realização: 0 anonimato dos respondentes, a representação estatística da distribuição dos resultados e o feedback de respostas do grupo para reavaliação nas rodadas subseqüentes (MARTINO, 1993). Tais reavaliações e trocas de informações são consideradas fundamentais para a obtenção de um grau satisfatório de convergência nas respostas, já que os respondentes podem assim rever suas posições anteriores frente à argumentação do grupo, assumindo assim uma posição de consenso (WRIGHT; GIOVINAZZO, 2000). O objetivo estritamente aplicado a tendências e eventos futuros vem sendo ampliado e atualmente a Técnica tem incorporado idéias e estratégias mais gerais para apoio à tomada de decisões e à definição de políticas, estabelecendo-se como Policy Delphi (Delphi de Políticas) (CASSIANI; RODRIGUES, 1996; WRIGHT; GIOVINAZZO, 2000).

Algumas têm sido as vantagens da técnica apontadas na literatura (WRIGTH, 1986): realizar previsões em situações de carência de dados históricos; analisar o problema por meio da consulta a um grupo de especialistas, possibilitando um volume maior de informações e o compartilhamento do nível de informação do 
membro melhor informado; conduzir a uma maior reflexão e cuidado nas respostas pelo fato de serem escritas; possibilitar que não haja influência de fatores como status acadêmico ou profissional já que as respostas são anônimas; reduzir fatores restritivos da dinâmica de grupo, como a supressão de posições minoritárias ou mesmo omissão de participantes; diminuir custos com deslocamento e reunir opiniões de vários indivíduos sem ter que reuni-los num mesmo local (CASSIANI; RODRIGUES, 1996). Outra vantagem destacada é possibilidade da presença de contradições nas opiniões dos diferentes grupos (PIOLA; VIANNA; VIVAS-CONSUELO, 2002).

As desvantagens apontadas (WRIGTH, 1986) são: selecionar amostras e analisar os resultados de modo estatisticamente não aceitável; excessiva dependência dos resultados em relação à escolha dos especialistas; possibilidade de forçar o consenso indevidamente; dificuldade de construção de um questionário sem perguntas ambíguas; longo período de realização do processo completo e custos elevados para a realização; problemas no retorno dos questionários e na identificação do grupo participante (CASSIANI; RODRIGUES, 1996).

\section{Oficinas de construção de indicadores e dispositivos de avaliação}

No campo da saúde mental, o termo "Oficina" tem sido bastante utilizado como referência ao trabalho com grupos, ganhando sentidos e usos diversos nos diferentes contextos em que são utilizadas. Segundo Tallemberg (2005), a etimologia da palavra Oficina vem de ofício (do latim officium) e expressa dever, em que o modo de fazer, além de transmitido artesanalmente de uns a outros, tem um sentido de compartilhamento, de experiência partilhada. Tal como sugere Vaisberg (2004), trabalhamos com a idéia de Oficinas como dispositivos profícuos para o desenvolvimento compartilhado de um ofício que fizesse sentido para os participantes e que lhes possibilitasse protagonizar a análise do contexto de que fazem parte. Não se tratou de, simplesmente, cumprir com a tarefa de produção de um material. Organizamos as Oficinas de modo que elas privilegiassem a criação coletiva, criação de um material que, além de responder às nossas interrogações de pesquisadores, mediasse um processo de construção de autoria, vivido pelos sujeitos da pesquisa. A seguir, apresentamos o processo de realização das oficinas e alguns exemplos de resultados por elas produzidos:

\section{Oficina de planejamento}

Primeiramente, organizamos uma Oficina de planejamento, denominada de "Oficina-mãe"6, cujo objetivo era planejar com diferentes atores as 10 Oficinas que viriam posteriormente. Para tanto, esta Oficina contou com a participação do grupo de pesquisa 
da Unicamp, alguns pesquisadores da UFF, o colegiado municipal de saúde mental, os gestores de CAPS, o comitê de gestão da pesquisa7 e o apoio de uma especialista em planejamento. Essa "Oficina-Mãe" construiu o formato a ser desenvolvido nas demais Oficinas, cujo objetivo foi a elaboração, em conjunto com os participantes da pesquisa, de indicadores e dispositivos de avaliação.

A "Oficina Mãe" foi estruturada da seguinte maneira: os participantes foram divididos igualitariamente em cinco subgrupos sendo que cada um destes ficou responsável por trabalhar um tema: 1) Concepção de CAPS; 2) Projeto terapêutico individual e práticas grupais; 3) Atenção à crise; 4) Formação profissional e sofrimento dos trabalhadores e 5) Gestão. O tema sobre as relações com a Rede permaneceu transversal a todos os grupos. Em cada subgrupo havia pelo menos uma dupla de pesquisadores auxiliando no processo de trabalho, além de uma especialista em planejamento, que não participou das etapas anteriores, com o papel de circular nos pequenos grupos apoiando a execução da tarefa.

Sintetizamos os núcleos argumentais de cada tema, apresentando-os na forma de "problemas", "controvérsias" e "soluções". Como já mencionamos, os núcleos argumentais são a reunião de todos os núcleos narrativos, isto é, a reunião de todos os argumentos de todos os grupos sobre determinado tema. Os núcleos argumentais considerados "problemas" foram aqueles identificados como uma situação não resolvida e de difícil solução pelos participantes. Foram classificados como controvérsias, aqueles núcleos argumentais que apontavam para opiniões opostas a respeito da mesma questão e foram classificados como soluções aqueles núcleos argumentais nos quais havia uma concordância a respeito de determinada questão.

Em relação ao tema Projeto Terapêutico Individual (PTI) são exemplos do material apresentado:

Problemas: há a necessidade de atenção constante à linha tênue do cuidado, pois existe o risco da desresponsabilização, sob pretexto do respeito à liberdade do paciente e, por outro lado, o risco do excesso de cuidado, que provoca controle e uma dependência tal, que o paciente não pode mais viver sem o serviço.

Controvérsias: segundo os usuários, em alguns CAPS o PTI é definido junto dos usuários e em outros apenas entre os profissionais.

Soluções: segundo os usuários, um usuário fala, um funcionário fala, e vai se formando o PTI. Depende da necessidade: alguns ficam no CAPS a maior parte do tempo, outros menos, pois a cabeça voltou a funcionar. Então, o tratamento funciona com funcionários que querem saber como o usuário está, orientam os remédios, vêem se é preciso ir até a família.

O material completo foi apresentado aos subgrupos que tinham como tarefa fazer a leitura e discussão dos núcleos argumentais e refletir a respeito de como estes dados qualitativos poderiam ser trabalhados 
nas Oficinas pelos participantes da pesquisa de modo que eles pudessem criar um modelo de trabalho que propiciasse, a partir deste material, a criação de indicadores e dispositivos avaliativos para o CAPS.

Os subgrupos também deveriam definir quais os outros atores seriam convidados para cada oficina, além das pessoas que estavam envolvidas no processo da oficina de planejamento. Definimos em plenária que as Oficinas seriam realizadas em duas etapas. $\mathrm{Na}$ primeira etapa seriam realizadas 5 oficinas, uma a respeito de cada tema e depois, repetido este processo na segunda etapa. Cada oficina contaria com cerca de 30 participantes por temática. Ao final do processo, teríamos realizado 10 oficinas, totalizando cerca de 300 participantes.

É importante salientar que já nesse momento de preparação das Oficinas, privilegiamos a participação dos diversos atores, de tal modo que os membros do grupo de pesquisa deslocavam-se até os serviços para com eles organizar questões de estrutura e conteúdo que envolviam o trabalho.

\section{Primeira etapa de oficina de Construção de Indicadores e Dispositivos Avaliativos}

Para cada temática acima enumerada, realizamos uma oficina, cujos participantes eram representantes de trabalhadores, gestores, usuários e familiares dos CAPS e do Colegiado de Saúde Mental, supervisores de CAPS bem como representantes de outros serviços da Rede, como SAMU, Unidades Básicas de Saúde, prontos socorros, Centros de Convivência, secretarias de cultura, esportes, habitação e transporte. Assim, para a oficina de atenção à crise, por exemplo, convidamos trabalhadores dos CAPS e UBS, gestores, usuários, familiares e representantes do SAMU e prontos socorros; já para a oficina de formação profissional e sofrimento dos trabalhadores, privilegiamos a presença de trabalhadores, gestores e supervisores dos CAPS. Portanto, para cada oficina convidamos diferentes representações segundo suas relações com o tema a ser discutido. Realizamos também um considerável aumento no número de familiares e usuários participantes em relação aos grupos focais, já que procuramos nos valer de uma técnica de criação de consenso que diversificasse e ampliasse a participação, com objetivo de radicalizar a pactuação dialógica entre os múltiplos grupos de interesse envolvidos.

Para a Oficina que trabalhou o tema Projeto Terapêutico Individual e Práticas grupais, por exemplo, foram convidados os 2 gerentes de CAPSIII da cidade, 2 apoiadores de Saúde Mental, 6 trabalhadores de CAPSIII (sendo um representante de cada serviço da cidade), 2 trabalhadores de UBS, 3 usuários de CAPSIII pertencentes a Conselhos de Saúde e 9 usuários não pertencentes a Conselho de 
Saúde, 2 trabalhadores de Centros de Convivência e 6 familiares de usuários de CAPS (sendo um representante por serviço).

Na primeira etapa, para cada Oficina, fizemos uma apresentação da pesquisa como um todo, a fim de atualizar quem não havia participado da pesquisa desde seu início, explicitando também quais eram nossos objetivos naquele momento. Em seguida, lemos junto com os participantes a relação de "problemas", "controvérsias" e "soluções" relativos ao tema da Oficina e elaborados a partir das narrativas dos grupos focais (portanto, se tratava de extratos de falas dos próprios sujeitos da pesquisa, agrupadas segundo os eixos temáticos). Após essa leitura conjunta, dividimos os participantes em dois grupos: um deles discutiria o material sintetizado sob a consigna problemas e controvérsias e selecionaria os temas considerados mais importantes (e, portanto, prioritários para merecer um indicador ou analisador de acompanhamento futuro). O outro grupo faria o mesmo com o material denominado soluções.

Foi necessário bastante cuidado dos pesquisadores em explicitar que nosso objetivo era a construção de indicadores e dispositivos de avaliação, a partir de um material elaborado com base na discussão anteriormente realizada nos grupos focais, constituídos com atores diretamente envolvidos com o processo de trabalho dos CAPS. Assim, não propúnhamos rediscutir as temáticas, realizando novos grupos focais, mas sim trabalhar a partir da discussão já existente, com a finalidade de construção dos indicadores e dispositivos de avaliação.

Em relação à temática $\mathrm{PTI}$, dos diversos núcleos argumentais apresentados foram escolhidos aqueles que os sujeitos entenderam merecer um monitoramento avaliativo:

- Algumas vezes, por conta do excesso de demanda e da escassez de recursos, o PTI acaba se reduzindo ao 'leque' do que há no serviço, não atendendo, "o que seria biopsicossocial, ou a reinserção social".

- Familiares não sabem exatamente o que é PTI. A equipe, geralmente, só fala a sigla, não explica o significado.

- A Equipe de Referência permite a discussão e a troca entre profissionais, propicia que a vinculação com os usuários se dê de diferentes maneiras, possibilita que se conheça melhor cada caso e protege a equipe contra alta rotatividade dos médicos. Em geral, cada equipe de referência tem um médico, psicólogos, enfermeiros, terapeutas ocupacionais, técnicos de enfermagem e é mais ou menos distribuída por micro-regiões do território do CAPS.

\section{Segunda etapa de oficina de Construção de Parâmetros e Dispositivos Avaliativos}

Privilegiamos que o intervalo entre as duas etapas de Oficinas não ultrapassasse quinze dias, pois apesar de buscar que os participantes tivessem um tempo para assimilar o trabalho desenvolvido na primeira etapa (podendo inclusive levar o material para seus serviços 
ou outros espaços de conversa), cuidamos para que esse período não fosse longo demais, diminuindo a implicação com a pesquisa e podendo prejudicar o trabalho da segunda etapa.

Na segunda etapa, realizamos outras 5 oficinas, divididas segundo as mesmas temáticas da etapa anterior e constituídas pelos mesmos participantes (30 pessoas por oficina). Não houve perda significativa de sujeitos de uma etapa para outra. Nesse momento, procedemos à elaboração de indicadores e analisadores em relação aos temas escolhidos no primeiro encontro, de modo que esses pudessem ser adotados para o monitoramento avaliativo.

Nesse contexto, as Oficinas objetivaram a devolutiva do material construído em campo, o incremento da participação dos diversos atores envolvidos na pesquisa, o processamento dos resultados da pesquisa e o estímulo à utilização destes pelos grupos de interesse, além de contribuir também para criação de consensos mínimos para continuidade do processo avaliativo e sua institucionalização.

São exemplos de indicadores construídos na Oficina que tratou do tema PTI:

- Porcentagem de pacientes para os quais o PTI inclui outros recursos da rede, que extrapolam o que é oferecido no CAPS.

- Porcentagem de trabalhadores que participam de alguma atividade em serviços for do CAPS (como apoio matricial, participação em fórum intersetorial, etc)

- porcentagem de pacientes que tiveram seu caso discutido no último ano. A equipe de referência discute, em média, pelo menos dois casos por semana?

- quantas discussões do PTI são feitas com a família, por ano?

Todas as oficinas construíram indicadores ou dispositivos através dos quais os CAPS podem ser avaliados ${ }^{8}$. Houve consenso em torno desses dispositivos e da necessidade de avaliação permanente, avaliação esta que requer um duplo respeito: por um lado, é necessário cumprir com as diretrizes da política nacional de saúde mental. Por outro lado, espera-se que as peculiaridades de cada serviço sejam sempre consideradas e debatidas.

Algumas questões envolveram discussões tensas, em que as diferenças de concepção e posicionamento político-teórico eram mais acirradas. Entre elas, estava a escolha por internar pacientes em hospitais psiquiátricos ou exclusivamente em hospitais gerais ou, ainda, em utilizar apenas os leitos-noite dos CAPS. Não chegamos a eleger a melhor das alternativas, mas foi consensual a necessidade de avaliação de cada caso e das circunstâncias em que a crise está sendo experimentada. Outro consenso se deu em torno da necessidade de envolver outros atores nessa decisão, além dos terapeutas que acompanham o caso e o profissional do SAMU, que regula as vagas. Assim, propôs-se a criação de um dispositivo de 
avaliação, composto por profissionais de vários CAPS que se reuniria para a discussão dos casos para os quais se pensava na internação.

De modo geral, os principais consensos construídos se deram em torno dos indicadores, bem como da constatação da necessidade de ampliação dos espaços coletivos de decisão (dentro e fora do CAPS), da revitalização daqueles já existentes (como Conselhos de Saúde), da intensificação da participação de familiares no cotidiano dos serviços e do alargamento da rede de relações do CAPS com outros serviços e setores da cidade.

\section{Discussão}

É variada a literatura científica que comprova a eficácia das técnicas de construção de consenso para as pesquisas qualitativas (CARLINECOTRIM, 1996; MORGAN, 1997; JONES; HUNTER, 1995). No entanto, verificamos que mudanças nessas técnicas, tal como realizamos por meio das Oficinas acima descritas, podem contribuir para o aprofundamento das reflexões acerca da pesquisa qualitativa e do seu alcance. No processo da pesquisa aqui relatado, as Oficinas foram tomadas como momento potencializador da participação dos diferentes atores, de compartilhamento de saberes e práticas em avaliação.

Consideramos que uma das suas potencialidades foi justamente 0 aumento numérico de atores pertencentes ao contexto do objeto estudado (passamos de aproximadamente 100 sujeitos nos grupos focais para cerca de 300 nas oficinas), bem como a radicalização da diversidade deles. Diversidade que não se baseou apenas na participação de um grupo de especialistas, tal como acontece nas tradicionais técnicas de consenso, mas na inclusão de vários grupos de interesse, uma vez que tinham importantes ligações com a realidade estudada.

Consequentemente, a construção de consensos sobre os indicadores e analisadores teve que se dar em torno do debate entre múltiplas diferenças, tais como níveis educacionais, categorias profissionais, posições sociais, condições econômicas, atributos de poder e vivências pessoais. Consideramos que a aposta na inclusão dos diferentes atores, ainda que não fossem reconhecidamente especialistas no assunto em avaliação, enriqueceu o processo, uma vez que os indicadores e dispositivos de avaliação construídos passaram a abranger questões ligadas a essa diversidade de atores, os quais tinham diferentes envolvimentos com o objeto de estudo.

Além disso, outro resultado importante dessa conformação das Oficinas diz respeito ao próprio processo de pesquisa e aos sujeitos do estudo. Ao serem colocados em contato não apenas com seus pares, mas também com pessoas de diferentes posições sociais, eles tiveram que se ouvir em suas diferenças e também que se posicionar, firmando e defendendo avaliações. Ao fazê-lo, precisaram tornar mais 
claras as suas opiniões, revê-las e aprofundá-las, o que acarretou em interessante processo político de construção de uma autoria coletiva sobre os modos de avaliação da realidade de trabalho, modos que, é claro, incluíam desejos, interesses, necessidades e perspectivas acerca dessa realidade.

Nesse sentido, salientamos que a potencialidade das Oficinas residiu na sua capacidade de expor diferenças não apenas para conversar, como também para construir os indicadores e dispositivos avaliativos. Tal potencialidade, entretanto, esteve condicionada pela estratégia adotada pelas Oficinas de incluir atores diferentes, colocá-los em debate, permitindo a expressão das discordâncias e dos dissensos e, finalmente, solicitar-lhes a construção de indicadores e dispositivos de avaliação. Note-se que tal construção marca uma diferença fundamental com aquela obtida por meio das Técnicas de Grupo Nominal e Delphi: ela produz um consenso em torno do parâmetro avaliativo construído, mas um consenso composto por diferenças de perspectivas e, inclusive, por dissensos apresentados e discutidos pessoalmente entre os atores envolvidos.

Aqui cabe uma explicitação sobre a composição de consenso e a produção de verdades, as relações de poder e o posicionamento dos grupos de interesse no campo. Em alguns momentos dos debates, a presença de usuários e familiares, ou de membros de outras equipes que não de CAPS operou algumas rachaduras em posicionamentos fechados, e às vezes até apresentados como polares. Como exemplo: profissionais de nível universitário de dois CAPS diferentes argumentavam a favor e contra a possibilidade de assistir a crise no domicilio, embrenhando-se por sofisticado repertório clínico psicanalítico, quando uma familiar disse: "meu irmão esquizofrênico mora sozinho com minha mãe que é idosa e eu não tenho nenhuma dúvida que quando ele não está bem é ótimo que o pessoal do CAPS vá lá em casa...". A legitimidade dessa fala baseada numa experiência pessoal produziu um primeiro momento de estranhamento e silêncio, seguido pela elaboração de um indicador a respeito da temática da crise.

Objetivamos, com essa reflexão, destacar o caráter construtivo das Oficinas. Uma construção obtida a partir da discussão, da revisão de posicionamentos, de encontros e discordâncias. Entendemos que a possibilidade de tal reposicionamento, marcado pelo encontro entre distintas perspectivas acerca da realidade avaliada, foi um efeito da aposta no protagonismo dos participantes no processo de avaliação. Esses foram convocados a deixar a posição de "avaliados" ou "pesquisados", para tornarem-se avaliadores do processo e criadores de indicadores e dispositivos de avaliação a serem utilizados para a continuidade do processo avaliativo.

Note-se que, com essas possibilidades construtivas, as Oficinas caminharam na direção da superação da dicotomia sujeito-objeto, 
marcadas por produções do pesquisador sobre o campo e não com os sujeitos. Com essa superação, entendemos ter alcançado um dos objetivos da pesquisa qualitativa: potencializar saberes excluídos, garantindo e legitimando a produção de conhecimento de usuários, trabalhadores, gestores dos serviços de saúde (ALVAREZ; PASSOS; CARVALHO; CÈSAR, 2008).

Além disso, o trabalho com as Oficinas foi ao encontro das reflexões propostas por Furtado (2008), para quem a avaliação deve se dar não por aplicação de valores sobre uma determinada realidade, mas por construção coletiva de valores comuns, com os quais a realidade, ela própria, se modifica. Trata-se, então, de um processo no qual os valores com os quais se avalia não podem restar no âmbito em que alguns - os avaliadores - detêm a decisão sobre aquilo a ser usado nos julgamentos das práticas. Nesse sentido, reiteramos a vantagem das Oficinas em não trabalhar apenas com especialistas hegemonicamente reconhecidos, incluindo grupos de interesse que experimentam cotidianamente o processo avaliado, embora não tenham formulações acadêmicas estruturadas acerca deles.

Contudo, um dos possíveis problemas associado às Oficinas diz respeito, justamente, à sua capacidade de incluir as diferentes vozes nos indicadores e analisadores consensuados. Sabemos que há jogos de poder inerentes ao convívio entre diferentes condições sociais, econômicas, educacionais, de acesso à linguagem, entre outras. Nesse sentido, o leitor poderia nos questionar acerca da capacidade das Oficinas em incluir os usuários, tanto quanto os médicos, na construção do parâmetro avaliativo sobre o acompanhamento das situações de crise no serviço, por exemplo.

Certamente, trata-se de uma fragilidade que, conforme vimos anteriormente, estaria resolvida na técnica Delphi, uma vez que os participantes não se encontram pessoalmente e desconhecem a identidade das respostas com que interagem, mas perderíamos assim o efeito de produção de verdade ao qual nos referimos acima. $\mathrm{Na}$ nossa pesquisa, ao optarmos pelas vantagens do encontro pessoal, precisamos contar com a habilidade dos coordenadores das Oficinas em incluir os diferentes participantes na discussão. Porém, outros recursos talvez também pudessem ser adotados em futuros estudos, tais como a adoção de um número maior de participantes que historicamente tenham tido menor acesso à formação de consensos, como o de usuários e familiares. Ao levar para as Oficinas o material produzido nos Grupos Focais e processado pelo grupo de pesquisa, solicitando que os participantes trabalhassem sobre ele, o fizemos norteados pela hermenêutica como postura metodológica, de tal modo que buscamos rever essa produção com diversidade de olhares, a fim de ampliar nossa compreensão acerca dela. 


\section{À guisa de conclusão}

Em se tratando de pesquisas avaliativas, a utilidade dos resultados é sempre um assunto de relevância. Sendo assim, pretendemos contribuir para a avaliação do serviço e, com os resultados, subsidiar a tomada de decisões para o melhoramento das práticas nos serviços pesquisados. Nossa atenção se deteve não apenas nos resultados, mas também nos movimentos produzidos ao longo do processo avaliativo, destacando-se aí a dimensão ético-política da pesquisa.

Parece-nos claro que inovações em termos de desenhos participativos e construtivistas, como as propostas pelos estudos de quarta geração demandar-nos-ão também inovações nas técnicas de consenso. Assim a vinda à tona de conhecimentos e experiências nem sempre valorizados pela produção acadêmica, porém relevante para a implementação de políticas públicas e a melhora dos serviços públicos de saúde parece-nos fundamental.

Procuramos honrar esse compromisso ético-político sem desistir do rigor e do debate com as técnicas consagradas. Esperamos que compartilhar esta experiência estimule outros grupos de investigação a testar suas próprias inovações, contribuindo para uma ciência viva, criativa e não somente prescritiva de metodologias prontas.

\section{Referências Bibliográficas}

ALVAREZ, A. P.; PASSOS, E. C.; CARVALHO, H. F. de; CÉSAR, J. M.; GONÇALVES, L. L. M.; DE BARROS, R. B. As oficinas como espaço do protagonismo dos sujeitos no processo de avaliação. In: ONOCKO CAMPOS, R. T; FURTADO J. P.; PASSOS, E.; BENEVIDES, R. (Orgs.). Pesquisa avaliativa em saúde mental: desenho participativo e efeitos de narratividade. São Paulo: Aderaldo e Rothschild, 2008, p. 300-318

CAMPOS, G. W. S. Um método para análise e co-gestão de coletivos. São Paulo: Hucitec, 2000.

Produção de conhecimento, avaliação de políticas públicas em saúde mental: notas reflexivas. In: ONOCKO CAMPOS, R. T.; FURTADO J. P.; PASSOS, E.; BENEVIDES, R. (Orgs.). Pesquisa avaliativa em saúde mental: desenho participativo e efeitos de narratividade. São Paulo: Aderaldo e Rothschild, 2008, p. 97-102.

CARLINE-COTRIM, B. Potencialidades da técnica qualitativa grupo focal em investigações sobre abuso de substâncias. Revista de Saúde Pública, São Paulo, v.30, n.3, 1996, p. 285-293.

CASSIANI, S. H. B.; RODRIGUES, L. P. A. Técnica de Delphi e a técnica de grupo nominal como estratégiaa de coleta de dados das pesquisas em Enfermagem. Acta Paulista de Enfermagem, v. 9, n. 3, p. 76-83, maio/jun. 1996.

FIGUEIREDO, M.; VIANNA, D. A rede de saúde de Campinas (SP): peculiaridades e inovações. In: ONOKCO CAMPOS, R. T. FURTADO, J. 
P.; PASSOS, E.; BENEVIDES, R. (Orgs.). Pesquisa avaliativa em saúde mental: desenho participativo e efeitos de narratividade. São Paulo: Aderaldo \& Rothschild, 2008, p. 155-162.

FURTADO, J. P. Avaliação como dispositivo. 2001. 243 f. Tese (Doutorado em Saúde Coletiva) - Faculdade de Ciências Médicas. Universidade Estadual de Campinas, Campinas.

FURTADO, J. P.; ONOCKO CAMPOS, R. T. A transposição das políticas de saúde mental no Brasil para a prática nos novos serviços. Revista Latino-americana de Psicopatologia Fundamental, v. VIII, n. 1, p. 109-122, mar. 2005.

- O percurso da pesquisa avaliativa de uma rede de Centros de Atenção Psicossocial: entre a saúde coletiva e a saúde mental. In: ONOCKO CAMPOS, R. T.; FURTADO J. P.; PASSOS, E.; BENEVIDES, R. (Orgs.). Pesquisa avaliativa em saúde mental: desenho participativo e efeitos de narratividade. São Paulo: Aderaldo \& Rothschild, 2008, p.189-208.

GADAMER, H. G. Verdade e método. Traços fundamentais de uma hermenêutica filosófica. Petrópolis, RJ: Vozes, 1997.

GUBA, E. G.; LINCOLN, Y. S. Fourth generation evaluation. Newbury Park: Sage Publications, 1989.

JONES, J.; HUNTER, D. Qualitative research: consensus methods for medical and health services research. British Medical Journal, v. 311, n.7001, p.376-380, 1995.

Usando o Delfos e a técnica de grupo nominal na pesquisa em serviços de saúde. In: POPE, C.; MAYS, N. (Orgs.). Pesquisa qualitativa na atenção à saúde. Tradução de Ananyr Porto Fajardo. Porto Alegre: Artmed, 2005, p. 51-60.

MARTINO, J. P. Technological forescasting for decision making. 3. ed. New York: Mc Graw-Hill Inc., 1993.

MENDES, W.; TRAVASSOS, C.; MARTINS, M.; MARQUES, P. M. Adaptação dos instrumentos de avaliação de eventos adversos para uso em hospitais brasileiros. Revista Brasileira de Epidemiologia, São Paulo, v. 11, n.1, p. 55-66, mar. 2008.

MIRANDA， L.; FIGUEIREDO, M. D. ; FERRER, A. L. ; ONOCKO CAMPOS, R. T. Os grupos focais aos grupos focais narrativos: uma descoberta no caminho da pesquisa. In: ONOKCO CAMPOS, R. T.; FURTADO, J. P. ; PASSOS, E. ; BENEVIDES, R. (Orgs.). Pesquisa avaliativa em saúde mental: desenho participativo e efeitos de narratividade. São Paulo: Aderaldo \& Rothschild, 2008, p. 249-277.

MORGAN, D. L. Focus groups as qualitative research. Thousand Oaks: Sage Publications; 1997.

MURPHY, M. K.; BLACK, N. A.; LAMPING, D. L.; MCKEE, C. M.; SANDERSON, C. F.; ASKHAM, J.; MARTEAU, T. Consensus development methods, and their use in clinical guideline development. Health Technology Assessment. v. 2, n. 3, p. 88, mar. 1998. 
ONOCKO CAMPOS, R. T. Clínica: a palavra negada - sobre as práticas clínicas nos serviços substitutivos de saúde mental. Revista Saúde em Debate, Rio de Janeiro, v. 25, n. 58, p. 98-111, maio/ago. 2001. O encontro trabalhador-usuário na atenção à saúde. Uma contribuição da narrativa psicanalítica ao tema do sujeito na saúde coletiva. Revista Ciência e Saúde Coletiva, v. 10, n. 3. p. 573583, jul./set. 2005.

- O exercício interpretativo. In: ONOCKO CAMPOS, R. T.; FÜRTADO J. P.; PASSOS, E.; BENEVIDES, R. (Orgs.). Pesquisa avaliativa em saúde mental: desenho participativo e efeitos de narratividade. São Paulo: Aderaldo \& Rothschild, 2008, p. 278-299.

ONOCKO CAMPOS, R. T.; FURTADO, J. P. Narrativas: utilização na pesquisa em saúde. Revista de Saúde Pública, São Paulo, v. 42, n. 6, p. 1090-1096, dez. 2008.

PASSOS, E.; SOUZA, T. P.; AQUINO, P. R.; BARROS, R. A. Processo coletivo de construção de instrumentos de avaliação: aspectos teóricos e metodológicos sobre dispositivos e indicadores processuais. In: ONOKCO CAMPOS, R. T.; FURTADO, J. P. ; PASSOS, E. ; BENEVIDES, R. (Orgs.). Pesquisa avaliativa em saúde mental: desenho participativo e efeitos de narratividade. São Paulo: Aderaldo e Rothschild, 2008, p. 375-397.

PIOLA, S. F., VIANNA, S. M., VIVAS-CONSUELO, D. Estudo Delphi: atores sociais e tendências do sistema de saúde brasileiro. Cadernos de Saúde Pública, Rio de Janeiro, v. 18 (suplemento), p.181-190, maio/jun. 2002.

RICOUER, P. I nterpretação e ideologias. Rio de Janeiro: Francisco Alves Editora; 1990.

Tempo e narrativa. Tomo I. Campinas: Papirus, 1994.

TALLLEMBERG, C. Fazendo da clínica uma oficina: modos de experimentação e produção social em saúde mental. Revista Eletrônica Academus: Revista Científica de Saúde/Secretaria Municipal de Saúde do Rio de Janeiro, v. IV, n. 1, p. 18 -38, jan./mar. 2005.

VAISBERG, T. A. Da questão do método à busca do rigor. In:

Ser e fazer: enquadres diferenciados na clínica winnicottiana. Aparecida (S.P): Idéias e Letras, 2004, p. 119-129.

WRIGTH, J. T. C. A técnica Delphi: Uma ferramenta útil para o planejamento do Brasil? In: ENCONTRO BRASILEIRO DE PLANEJ AMENTO EMPRESARIAL - "Como planejar 86", III, 29-29 nov. 1985. Anais. São Paulo: SPE - Sociedade Brasileira de Planejamento Empresarial, 1986, p. 199-207.

WRIGTH, J. T. C; GIOVINAZZO, R. A. Delphi - uma ferramenta de apoio ao planejamento prospectivo. Caderno de Pesquisas em Administração, São Paulo, v. 01, n. 12, p.54-65, 2000. jul./set. 2000. 


\section{Endereço para correspondência}

Rosana Teresa Onocko Campos

Rua Tessália Vieira Camargo, 126, Cidade Universitária "Zeferino Vaz", Caixa Postal 6111, CEP 13083-970, Campinas-SP, Brasil.

Endereço eletrônico: rosanaoc@mpc.com.br

Lilian Miranda

Rua Tessália Vieira Camargo, 126, Cidade Universitária "Zeferino Vaz", Caixa Postal 6111, CEP 13083-970, Campinas-SP, Brasil.

Endereço eletrônico: limiranda78@hotmail.com

Carlos Alberto Pegolo da Gama

Rua Tessália Vieira Camargo, 126, Cidade Universitária "Zeferino Vaz", Caixa Postal 6111, CEP 13083-970, Campinas-SP, Brasil.

Endereço eletrônico: carlosgama@terra.com.br

Ana Luiza Ferrer

Rua Tessália Vieira Camargo, 126, Cidade Universitária "Zeferino Vaz", Caixa Postal 6111, CEP 13083-970, Campinas-SP, Brasil.

Endereço eletrônico: aluizaferrer@ig.com.br

Alberto R. Diaz

Rua Tessália Vieira Camargo, 126, Cidade Universitária "Zeferino Vaz", Caixa Postal 6111, CEP 13083-970, Campinas-SP, Brasil.

Endereço eletrônico: algiova@gmail.com

Laura Gonçalves

Rua Tessália Vieira Camargo, 126, Cidade Universitária "Zeferino Vaz", Caixa Postal 6111, CEP 13083-970, Campinas-SP, Brasil.

Endereço eletrônico: lauralmg@terra.com.br

Thiago Lavras Trapé

Rua Tessália Vieira Camargo, 126, Cidade Universitária "Zeferino Vaz", Caixa Postal 6111, CEP 13083-970, Campinas-SP, Brasil.

Endereço eletrônico: thitrape@yahoo.com.br

Recebido em: 31/07/2009

Aceito para publicação em: 18/11/2009

Acompanhamento do processo editorial: Deise Mancebo, Marisa Lopes da Rocha e Roberta Romagnoli

\section{Notas}

* Médica, Doutora em Saúde Coletiva

**Psicóloga, membro do Grupo de pesquisa "Saúde Coletiva e Saúde Mental: interfaces"

***Psicólogo, membro do Grupo de pesquisa "Saúde Coletiva e Saúde Mental: interfaces"

****Terapeuta Ocupacional, membro do Grupo de pesquisa "Saúde Coletiva e Saúde Mental: interfaces"

*****Psicólogo, membro do Grupo de pesquisa "Saúde Coletiva e Saúde Mental: interfaces"

******Psicóloga, membro do Grupo de pesquisa "Saúde Coletiva e Saúde Mental: interfaces"

*******Psicólogo, membro do Grupo de pesquisa "Saúde Coletiva e Saúde Mental: interfaces"

${ }^{1}$ Consultar: <http://www.fcm.unicamp.br/grupos/saude_mental>. 
${ }^{2}$ Edital MCT-CNPq/ MS-SCTIE-DECIT / CT-Saúde - n. 07/2005 - Esta pesquisa foi aprovada e financiada pelo Conselho Nacional de Pesquisa, Cnpq.

${ }^{3}$ Campinas foi uma das pioneiras na adoção e criação dos modelos de atenção à saúde mental. A rede de assistência de saúde mental do município mostra-se bastante ampla e diversificada. Está organizada em vários níveis de atenção e conta com serviços básicos, serviços ambulatoriais especializados, serviços hospitalares, de urgência e emergência e serviços próprios da rede substitutiva ao hospital psiquiátrico. Para mais informações, FIGUEI REDO; VIANNA (2008).

4 Consideramos núcleos narrativos as argumentações presentes nas narrativas produzidas por cada grupo de interesse a respeito das diferentes temáticas. Consideraremos como núcleos argumentais o conjunto de argumentações produzidas por todos os grupos de interesse a respeito das diferentes temáticas.

5 Para mais informações, ONOCKO CAMPOS (2008).

${ }^{6}$ Para mais informações, ALVAREZ A. P.; PASSOS, E.; C.; CARVALHO, H F. de; CÉSAR, J. M.; GONÇALVES, L. L. M.; DE BARROS, R. B.(2008)

7 O Comitê de gestão da pesquisa foi criado com objetivo de operacionalizar o campo. Composto por um representante de cada CAPS III, essas pessoas foram encarregadas de ser o elo de comunicação entre os equipamentos e os pesquisadores, já que a literatura refere certa dificuldade na convocação das pessoas para participação nos grupos. Para mais informações, RODRIGUES, A. C; BEDIN, B. V; SILVA, D. L. S; FERIGATO, S; TAVARES, T. C. O comitê de pesquisa como articulador entre a Universidade e a rede de Caps de Campinas. In: ONOCKO CAMPOS, R. T; FURTADO, J. P.; PASSOS, E ; BENEVIDES, R. (Orgs.). Pesquisa avaliativa em saúde mental: desenho participativo e efeitos de narratividade. São Paulo: Aderaldo \& Rothschild, 2008, p.337-343.

${ }^{8}$ Para mais informações, PASSOS, E.; SOUZA, T. P.; AQUINO, P. R.; BARROS, R. A. (2008). 